Check for updates

Cite this: J. Mater. Chem. C, 2017, 5, 10861

Received 16th June 2017, Accepted 26th September 2017

DOI: $10.1039 / c 7 t c 02699 d$

rsc.li/materials-c

\section{Probing the local structure of the near-infrared emitting persistent phosphor $\mathrm{LiGa}_{5} \mathrm{O}_{8}: \mathrm{Cr}^{3+} \uparrow$}

\author{
Olivier Q. De Clercq, (D) ${ }^{\text {ab }}$ Lisa I. D. J. Martin, (D) ${ }^{a b}$ Katleen Korthout, (D) ab \\ Jevgenij Kusakovskij, (D) ${ }^{c}$ Henk Vrielinck (D) ${ }^{c}$ and Dirk Poelman (D)*ab
}

\begin{abstract}
Near-infrared emitting persistent phosphors have promising applications in the field of in vivo medical imaging. In this paper, we prepared the persistent phosphor $\mathrm{LiGa}_{5} \mathrm{O}_{8}: \mathrm{Cr}^{3+}(\mathrm{LGO}: \mathrm{Cr}$ ) which exhibits emission in the tissue transparency window and shows afterglow for multiple hours after excitation. Addition of Si or Ge improves the persistent luminescence. X-ray diffraction and electron microscopy, coupled with elemental analysis, revealed that there is a maximum amount of Si or Ge that can be incorporated in the host. Via X-ray absorption spectroscopy and electron paramagnetic resonance experiments, we studied the local environment of chromium in the LGO spinel host. The presence of both $\mathrm{Cr}^{3+}$ and $\mathrm{Cr}^{4+}$ on octahedral sites in LGO was confirmed. Electron paramagnetic resonance showed that $\mathrm{Cr}^{3+}$ resides in a rhombically distorted octahedral lattice site and that the $\mathrm{Cr}^{3+}$ local environment is sensitive to variation in point defects in the surrounding.
\end{abstract}

\section{Introduction}

Persistent phosphors are luminescent materials that keep emitting light long after the optical excitation has stopped. Since the discovery of the efficient green emitting phosphor $\mathrm{SrAl}_{2} \mathrm{O}_{4}: \mathrm{Eu}$,Dy by Matsusawa et al. in 1996, these materials have found uses in a wide range of applications such as toys, displays and safety signage. ${ }^{1-4}$ In 2007 , Le Masne de Chermont and coworkers showed in their proof-of-principle experiments that near-infrared emitting long persistent phosphors (NIR LPP) can also be used in bio-imaging applications as an alternative to radioactive probes. ${ }^{5}$ This application requires an emission spectrum in a wavelength region where absorption and scattering of light by blood and tissue components is minimal. Two wavelength ranges were identified: one between 650 and $950 \mathrm{~nm}$, and one between 1000 and $1350 \mathrm{~nm}$. These are known as the first and second tissue transparency window, respectively. ${ }^{6}$

Persistent phosphors have several advantages with regard to this application, in comparison with other optically active particles, such as upconversion nanoparticles, quantum dots or organic fluorophores. ${ }^{7-10}$ For instance, autofluorescence effects can be greatly reduced, which enhances the signal to

\footnotetext{
${ }^{a}$ LumiLab, Department of Solid State Sciences, Ghent University, Ghent, Belgium. E-mail:Dirk.Poelman@UGent.be

${ }^{b}$ Center for Nano- and Biophotonics (NB-Photonics), Ghent University, Ghent, Belgium

${ }^{c}$ EMR group, Department of Solid State Sciences, Ghent University, Ghent, Belgium

$\dagger$ Electronic supplementary information (ESI) available. See DOI: 10.1039/c7tc02699d
}

background ratio of measurements. Local heating effects as a consequence of high-power laser excitation, which is typically needed for upconversion, can also be avoided.

First generation NIR LPP were based on $\mathrm{Eu}^{2+}$ emission, ${ }^{11,12}$ as the europium ion shows the most efficient visible light persistent luminescence, in phosphors such as $\mathrm{SrAl}_{2} \mathrm{O}_{4}$ :Eu,Dy (green), $\mathrm{Sr}_{2} \mathrm{MgSi}_{2} \mathrm{O}_{7}$ :Eu,Dy (blue) and $\mathrm{CaAl}_{2} \mathrm{O}_{4}$ :Eu,Nd (violetblue). However, the $\mathrm{Eu}^{2+}$ emission needs to be significantly redshifted if one wants to use the LPP for imaging purposes. This limits the types of host that can be used for medical imaging purposes. Therefore, transition metals such as manganese or chromium that have longer emission wavelengths in a large variety of hosts have been investigated as alternative luminescent centers for this application. ${ }^{7}$

Second generation NIR LPP based on broad $\mathrm{Cr}^{3+}$ emission around $700 \mathrm{~nm}$ have gained interest since the development of Cr-doped $\mathrm{ZnGa}_{2} \mathrm{O}_{4}$ (ZGO:Cr) and other similar gallate hosts. ${ }^{7,8,13}$ The afterglow efficiency of ZGO:Cr can be greatly enhanced by incorporation of Ge ions. ${ }^{14-17}$ A model for the mechanism of persistent luminescence in ZGO:Cr has been developed throughout the years by a combined analysis of data obtained via different techniques. ${ }^{18-21}$ The currently accepted model attributes the afterglow behavior to the interaction between the $\mathrm{Cr}^{3+}$ ion and nearby anti-site defects in ZGO spinel.

In this paper, we investigate the structural properties of the persistent phosphor $\mathrm{LiGa}_{5} \mathrm{O}_{8}: \mathrm{Cr}^{3+}$ (LGO:Cr) that was reported to exhibit very efficient NIR persistent luminescence. ${ }^{22}$ The LGO:Cr phosphor has excellent properties for potential bioimaging applications, as it combines emission around $718 \mathrm{~nm}$ with good chemical stability in water and low toxicity. ${ }^{22,23}$ 
The overall structure of the LGO host was investigated using X-ray diffraction (XRD), and the effects of the addition of Si(Iv) and Ge(Iv) were studied. The latter tetravalent ions introduce charge mismatch in the lattice, which promotes the formation of compensating defects. The resulting defects were found to strongly increase the persistent luminescence time and intensity of LGO:Cr. Extended X-ray absorption fine structure (EXAFS) and electron paramagnetic resonance (EPR) measurements were conducted to probe the local environment of the Cr ions. From the X-ray absorption near edge structure (XANES) analysis, $\mathrm{Cr}^{3+}$ and $\mathrm{Cr}^{4+}$ species were identified, both located in octahedrally coordinated sites of the spinel lattice. EPR was used to confirm the coordination of $\mathrm{Cr}^{3+}$, and allowed to study the deformation of the site upon incorporation of the tetravalent Si or Ge ions in the host.

The present work thus extends previous investigations on LGO:Cr by combining PL, EXAFS and EPR to investigate the dopant incorporation of the $\mathrm{Cr}$ ions in LGO. In addition, the effects of codoping LGO:Cr with tetravalent ions were studied for the first time.

\section{Experimental}

LGO:Cr powder samples were prepared via solid state reaction. Stoichiometric amounts of $\mathrm{Li}_{2} \mathrm{CO}_{3}$ (99.998\%, Alfa Aesar) and $\mathrm{Ga}_{2} \mathrm{O}_{3}$ (99.99\%, Alfa Aesar) were ground in an agate mortar and transferred to open alumina crucibles. Cr was added as $\mathrm{Cr}_{2} \mathrm{O}_{3}$ (99.97\%, Alfa Aesar) with a molar concentration of $1 \%$ with respect to the $\mathrm{Ga}$ content. $\mathrm{M}$ (Iv) ( $\mathrm{M}=\mathrm{Ge}$ or $\mathrm{Si}$ ) was added as $\mathrm{GeO}_{2}$ (99.999\%, Alfa Aesar) or $\mathrm{SiO}_{2}$ (99.9\%, Alfa Aesar), respectively, to the LGO spinel structure according to the substitution $\mathrm{LiGa}_{5-x} \mathrm{M}_{x} \mathrm{O}_{8}: \mathrm{Cr}^{3+}(x=0.2,0.4$ or 1$)$.

This starting mixture was heated in ambient air to $800{ }^{\circ} \mathrm{C}$ for $2 \mathrm{~h}$ in order to remove the carbon content in the precursors, and subsequently to $1300{ }^{\circ} \mathrm{C}$ for $4 \mathrm{~h}$ to form the final host. ${ }^{22}$ The employed heating rate was $300{ }^{\circ} \mathrm{C} \mathrm{h}^{-1}$.

Crystallographic phases of the obtained powders were checked using X-ray diffraction (XRD) with a Bruker D5000 $\theta-2 \theta$ diffractometer using $\mathrm{Cu}-\mathrm{K} \alpha$ radiation $(40 \mathrm{kV}, 40 \mathrm{~mA}$, $\lambda=0.154 \mathrm{~nm}$ ) and compared with literature data (PDF-2 database of the ICDD).

SEM-EDX measurements were performed using a Hitachi S-3400 N scanning electron microscope (SEM), equipped with a Thermo Scientific Noran 7 energy-dispersive X-ray detector (EDX).

Photoluminescence emission and excitation spectra of the samples were measured with a fluorescence spectrometer (FS920, Edinburgh Instruments) using a monochromated $450 \mathrm{~W}$ Xe-arc as the excitation source. Afterglow decay profiles were measured using a Centronic OSD100-5T silicon photodiode, calibrated in absolute radiometric units and connected to a Hamamatsu C9329 photosensor amplifier. Prior to afterglow measurements, the samples were excited for $5 \mathrm{~min}$ by $1000 \mathrm{~lx}$ light of an unfiltered xenon arc lamp.

EPR spectra were recorded at the $\mathrm{X}$ band frequency $(9.42 \mathrm{GHz}$ ) on a Bruker ESP300E spectrometer and at the Q band frequency
(34.0 GHz) on a Bruker Elexsys E500 spectrometer. All EPR spectra were recorded at room temperature and with a microwave power of typically $2 \mathrm{~mW}$.

EXAFS and XANES measurements were performed at the $\mathrm{Cr}$ K edge at the Dutch-Belgian beam line (DUBBLE, BM26A), ESRF, Grenoble, France. ${ }^{24,25}$ Reference measurements were done on $\mathrm{Cr}$ foil and pressed pellets of $\mathrm{Cr}_{2} \mathrm{O}_{3}$ (99.97\%, Alfa Aesar) and $\mathrm{CrO}_{2}$ (99.97\%, Alfa Aesar). All spectra were recorded at room temperature. The atomic positions in the first coordination shells of the Mn atom were fitted by means of the FEFF6 code using the Athena and Artemis software by Ravel and Newville. ${ }^{26}$

\section{Results and discussion}

\section{$\mathrm{X}$-ray diffraction and crystal structure}

Measured XRD patterns show a crystalline structure with good correspondence with the reference pattern for $\mathrm{LiGa}_{5} \mathrm{O}_{8}$ (ICSD file 076-0199) (Fig. 1). The lattice constant was determined as $a=8.208 \AA$ for the undoped powders, after correcting for small height displacements during the positioning of the sample holder. ${ }^{28}$ The reference pattern corresponds to a crystal with space group $P 4_{3} 32$, which is a derivative of the $F d 3 m$ space group of spinel crystals. ${ }^{29,30}$

LGO has a cubic crystal structure with four formula units in the unit cell. Oxygen anions form a sublattice with a fcc framework, with cations occupying interstitial sites. $1 / 8$ of the possible tetrahedral and $1 / 2$ of the possible octahedral cation lattice sites are occupied in an ordered fashion. Tetrahedral sites are occupied by $\mathrm{Ga}^{3+}$ on the $8 \mathrm{c}$ Wyckoff positions; the remaining $\mathrm{Ga}^{3+}$ occupies $12 \mathrm{~d}$ sites and $\mathrm{Li}^{+}$occupies $4 \mathrm{~b}$ sites. The resulting crystal structure is that of an inverse spinel, and $\mathrm{LiGa}_{5} \mathrm{O}_{8}$ can thus also be written as $\left(\mathrm{Ga}_{2}\right)_{\mathrm{T}}\left(\mathrm{LiGa}_{3}\right)_{\mathrm{O}} \mathrm{O}_{8}$, where the subscripts ' $\mathrm{T}$ ' and ' $\mathrm{O}$ ' refer to tetrahedral and octahedral coordination respectively. It was reported that there is a slight mixing between $\mathrm{Li}$ and $\mathrm{Ga}$ on their respective Wyckoff positions, resulting in a deviation from the ordered LGO crystal structure. ${ }^{29,31}$

$\mathrm{M}(\mathrm{Iv})(\mathrm{M}=\mathrm{Si}$ or $\mathrm{Ge}$ ) was added to the LGO spinel structure according to the substitution $\mathrm{LiGa}_{5-x} \mathrm{M}_{x} \mathrm{O}_{8}: \mathrm{Cr}^{3+}$. Note that this

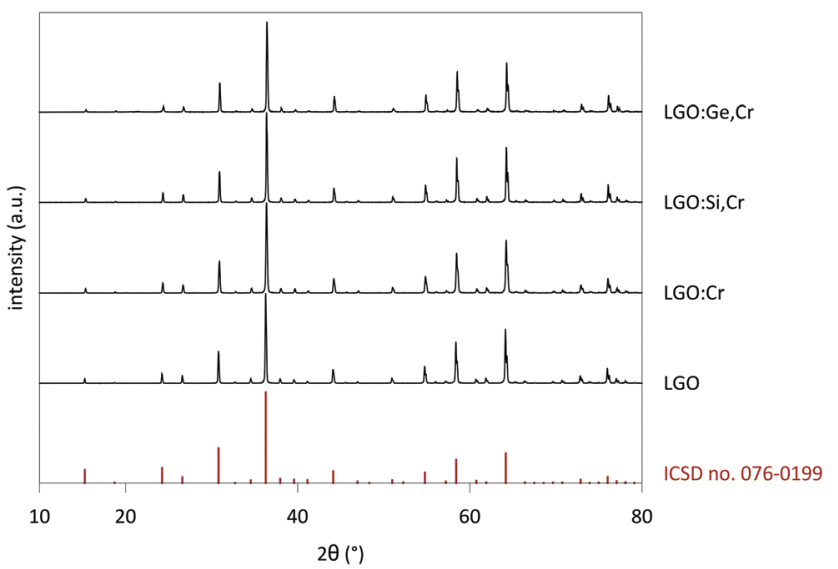

Fig. 1 XRD patterns of LGO, LGO:Cr, LGO:Si,Cr $(x=0.4), \mathrm{LGO}: \mathrm{Ge}, \mathrm{Cr}$ $(x=0.4)$ and reference pattern (ICSD file 076-0199). Samples are phasepure and correspond to the LGO reference for a $P 4_{3} 32$ space group. 
Table 1 Effective ionic radii (in Å) for tetra- and octahedral coordination of the cations present in the LGO lattice ${ }^{27}$

\begin{tabular}{llll}
\hline Element & Charge & Tetrahedral & Octahedral \\
\hline $\mathrm{Li}$ & $1+$ & 0.59 & 0.76 \\
$\mathrm{Si}$ & $4^{+}$ & 0.26 & 0.4 \\
$\mathrm{Cr}$ & $3+$ & - & 0.615 \\
& $4+$ & 0.41 & 0.55 \\
$\mathrm{Ga}$ & $3+$ & 0.47 & 0.62 \\
$\mathrm{Ge}$ & $4+$ & 0.39 & 0.53
\end{tabular}

formula unit is not charge neutral, so the substitution should lead to the formation of charge compensation point defects in the host that could be beneficial to the persistent luminescence.

Upon doping LGO with $\mathrm{Cr}^{3+}$ and M(Iv), the lattice parameter shifted a few $0.1 \%$, which is within the experimental errors of determination of the peak positions. The negligible change in $a$ indicates good incorporation of the dopants in the host, which is expected in view of the similar effective ionic radii of the cations (see Table 1). ${ }^{27}$ The lattice shifts were determined from the most intense peaks in the XRD patterns. Fig. S1-S3 (ESI $\dagger$ ) show detailed XRD patterns of selected LGO samples. Contrary to previously reported work, ${ }^{32}$ no impurities from a $\mathrm{LiGaO}_{2}$ side phase could be measured, which is an advantage of the solid state synthesis route employed here. When the M(Iv) content is increased to $x=1$ (Fig. S2-S3, ESI $\dagger$ ), traces of Si- and Ge-containing side phases appear in the measured XRD patterns, which suggests that there is a maximum $\mathrm{M}$ (IV) concentration that can be incorporated in the LGO lattice.

\section{SEM-EDX}

A discrepancy between nominal and actual $\mathrm{M}$ (Iv) content is evident from elemental analysis by SEM-EDX. The values from EDX were obtained via semi-quantitative analysis and not via the measurement of metal standards. Nominal and measured ratios of $\mathrm{M} / \mathrm{Ga}$ are provided in Table 2. The incorporation of $\mathrm{M}(\mathrm{IV})$ ions in the host is less than the nominal content for every value of $x$. The higher the M(Iv) content, the closer the measured ratios tend to the nominal ratios. However, elemental mapping images and XRD patterns show that an important fraction of $\mathrm{M}$ (IV) is not incorporated in LGO but present in side phases at higher concentrations. Fig. S4-S8

Table 2 Nominal and measured M/Ga ratios in LGO:Si,Cr and LGO:Ge,Cr. Data obtained via semi-quantitative EDX analysis. Concentrations measured using EDX have up to $20 \%$ relative error

\begin{tabular}{|c|c|c|c|}
\hline \multirow[b]{2}{*}{ LGO:Si,Cr } & \multicolumn{2}{|c|}{$\underline{\mathrm{Si} / \mathrm{Ga} \text { ratio }}$} & \multirow{2}{*}{$\frac{\mathrm{Cr} / \mathrm{Ga} \text { ratio }}{\mathrm{EDX}}$} \\
\hline & Nominal & EDX & \\
\hline$x=0.2$ & 0.04 & 0.018 & 0.012 \\
\hline$x=0.4$ & 0.09 & 0.037 & 0.011 \\
\hline \multirow[t]{2}{*}{$x=1$} & 0.25 & 0.181 & 0.015 \\
\hline & \multicolumn{2}{|c|}{ Ge/Ga ratio } & $\underline{\mathrm{Cr} / \mathrm{Ga} \text { ratio }}$ \\
\hline LGO:Ge,Cr & Nominal & EDX & EDX \\
\hline$x=0.2$ & 0.04 & 0.017 & 0.009 \\
\hline$x=0.4$ & 0.09 & 0.062 & 0.016 \\
\hline$x=1$ & 0.25 & 0.222 & 0.013 \\
\hline
\end{tabular}

(ESI $\dagger$ ) show the microstructure of several LGO powder samples. For M(Iv) codoped samples with $x=1$, it is clear that the Ga and M(Iv) EDX signals do not fully overlap, but are separated in the SEM image in specific regions. For codoped samples with $x=0.4$, there is a good overlap between the $\mathrm{Ga}$ and $\mathrm{M}$ (Iv) signals. Ge or Si are thus not fully incorporated in the LGO lattice at higher concentrations, despite the similar ionic radii of $\mathrm{Ge}^{4+}, \mathrm{Si}^{4+}$ and $\mathrm{Ga}^{3+}$. This is most probably a result of the nonisovalent $\mathrm{M}$ (Iv)-for-Ga(III) substitution, which requires charge compensation.

Measured chromium quantities correspond with nominal values within the error margins for semi-quantitative analysis for all samples, except $x=0.4$ samples. Concentrations were determined using EDX, with a relative error up to $20 \%$ (combined error due to sample inhomogeneity and intrinsic EDX errors). Due to the low Cr concentration in the sample, the EDX signal is low, but seems homogeneous. Mappings show no appreciable sign of clustering inside the material and the Cr signal nicely overlaps with other cation signals, indicating that $\mathrm{Cr}$ is wellincorporated.

\section{X-ray absorption spectroscopy}

The XANES spectra of the samples under study are displayed in Fig. 2. All spectra were aligned and merged using the Athena software to improve the $\mathrm{S} / \mathrm{N}$ ratio. $^{26}$

From these spectra two things can be observed: first, the preedge region of the measured samples shows a weak pre-edge which is typical for $\mathrm{Cr}$ ions in an octahedral coordination. XANES spectra of $\mathrm{Cr}$ ions which are tetrahedrally coordinated exhibit a single intense pre-edge peak which can be assigned to a dipole allowed transition of a $1 \mathrm{~s}$ electron to an unoccupied antibonding $\mathrm{t}_{2}{ }^{*}$ tetrahedral orbital. ${ }^{33}$ This intense peak is absent here.

Secondly, the absorption edge position of the measured samples is located between the absorption step of $\mathrm{Cr}_{2} \mathrm{O}_{3}$ and $\mathrm{CrO}_{2}$. This suggests the presence of $\mathrm{Cr}$ ions in both trivalent and tetravalent oxidation state.

In order to confirm these assumptions, EXAFS spectra were collected at the Cr $\mathrm{K}$ edge. The EXAFS spectra were analysed

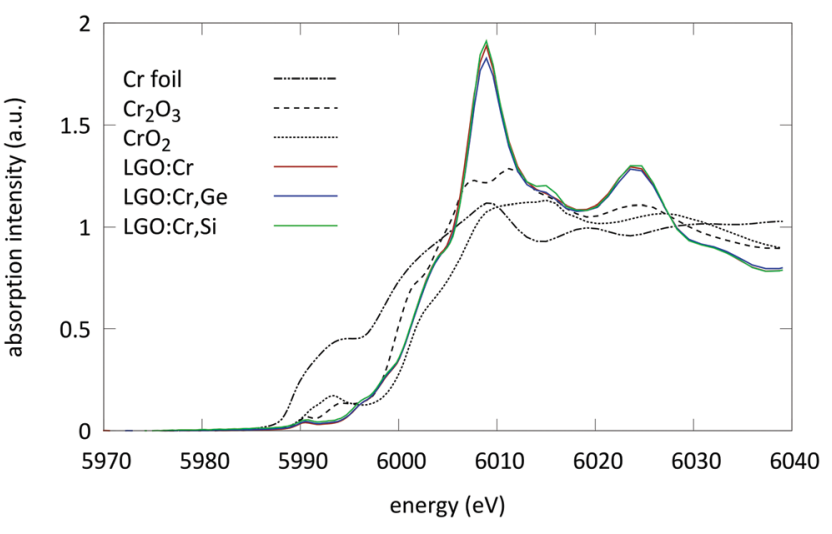

Fig. 2 XANES spectra of the LGO:Cr,M ( $x=0.4)$ samples measured the $\mathrm{Cr} \mathrm{K}$ edge together with the spectra of selected reference compounds (dashed lines): $\mathrm{Cr}$ foil (metallic), $\mathrm{Cr}_{2} \mathrm{O}_{3}$ (trivalent chromium on octahedral site) and $\mathrm{CrO}_{2}$ (tetravalent chromium on octahedral site). 


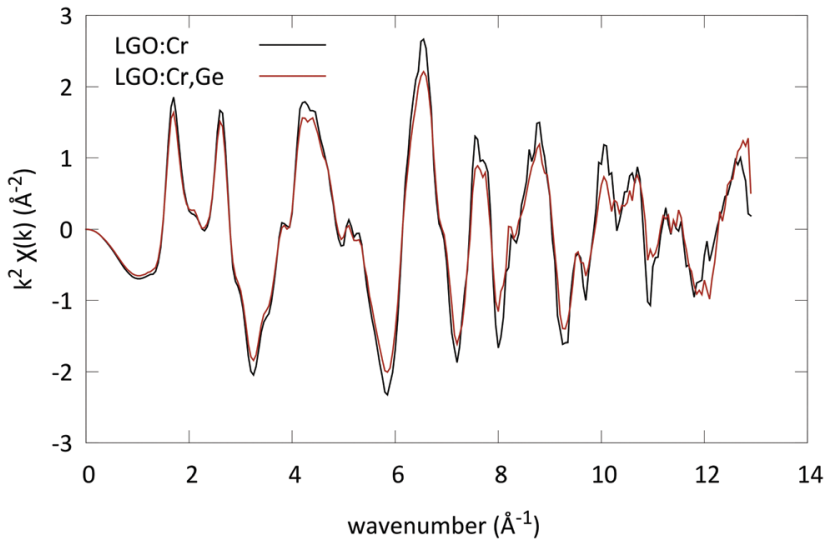

Fig. 3 EXAFS spectra of LGO:Cr and LGO:Ge,Cr $(x=0.4)$ converted to $k$-space. The spectra were recorded at the $\mathrm{Cr} \mathrm{K}$ edge, and accumulated for 4 scans.

using the Artemis software (Fig. 3 ). ${ }^{26}$ The spectra of the samples were analysed together using multiple data set fitting (MDS). This approach is often used if parameters are expected to be the same in different data sets. As such the number of parameters used can be maintained low.

For all spectra a Hanning window was used ( $k$-range: [2.683 $\left.\AA^{-1}, 10.84 \AA^{-1}\right]$, and $R$-range: $[1 \AA, 4.236 \AA]$ ). The structure of LGO was introduced to generate the scattering paths; both the octahedral and the tetrahedral Ga sites were used as possible locations for the $\mathrm{Cr}$ ion in the lattice. The scattering paths were calculated up to $4.5 \AA$ in $R$-space. Next to the possible substitution on two sites, the possible presence of two oxidation states was evaluated as well.

First all four virtual spectra (combining two coordinations and two valence states) were calculated to evaluate if one of the possibilities was preferential. The best match was found when the $\mathrm{Cr}$ ions occupy the octahedral $\mathrm{Ga}$ sites in the lattice. Subsequently the spectra were fitted using MDS with only this site and one oxidation state for the Cr ions (3+). An $R$-factor of $R=0.03$ was found (see Fig. 4).

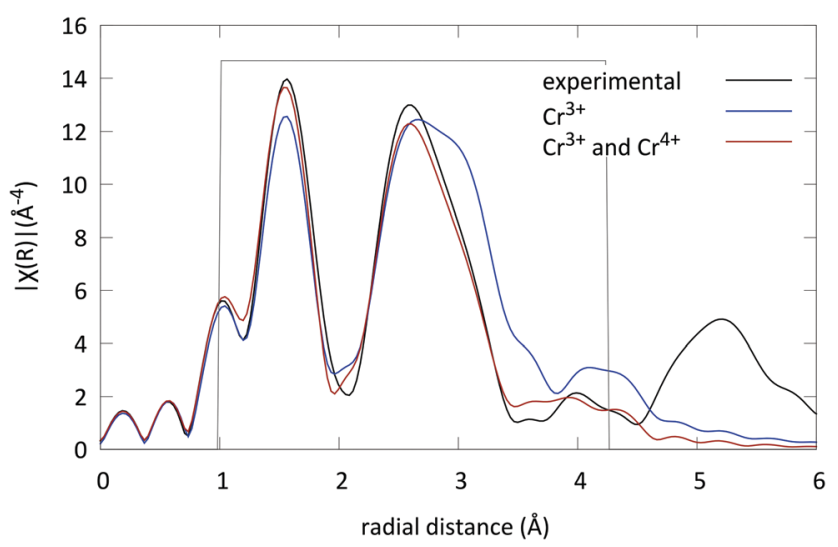

Fig. 4 Experimental EXAFS spectrum of LGO: $\mathrm{Cr}$ in $r$-space (black), fit of the EXAFS spectrum, taking only octahedral $\mathrm{Cr}^{3+}$ into account (blue), and the best EXAFS fit, with $\mathrm{Cr}^{3+}(69 \%)$ and $\mathrm{Cr}^{4+}(31 \%)$, both on octahedral sites in LGO (red).
Table 3 (top) Influence of $\mathrm{Cr}$ incorporation on the equilibrium distances with the nearest neighbours. $R(\mathrm{Axx})$ is the equilibrium distance as derived from the XRD pattern; $\Delta R(\mathrm{Axx})$ is the change as derived from the EXAFS spectra. (bottom) Debye-Waller factors for each type of scattering path, i.e. the parameter is only dependent on the type of scatterer

\begin{tabular}{lcc}
\hline & $\mathrm{Cr}^{3+}(\%)$ & $\mathrm{Cr}^{4+}(\%)$ \\
\hline$\Delta R(\mathrm{O} 21) / R(\mathrm{O} 21)$ & $-4.03(11)$ & $-4.41(26)$ \\
$\Delta R(\mathrm{O} 22) / R(\mathrm{O} 22)$ & $5.21(28)$ & $-1.48(52)$ \\
$\Delta R(\mathrm{O} 23) / R(\mathrm{O} 23)$ & $-1.35(19)$ & $-2.94(47)$ \\
$\Delta R(\mathrm{Ga11}) / R(\mathrm{Ga} 11)$ & $1.945(53)$ & $-0.62(12)$ \\
$\Delta R(\mathrm{Ga} 21) / R(\mathrm{Ga} 21)$ & $0.01(4)$ & $3.57(10)$ \\
\hline & Oxygen & Gallium \\
\hline$\sigma^{2}$ & $0.00456(24)$ & $0.00103(21)$
\end{tabular}

Subsequently the presence of another $\mathrm{Cr}$ absorber on the octahedral Ga site was introduced. The fit significantly improved and an energy shift of $0.82 \mathrm{eV}$ was found. This energy shift indicates a different oxidation state for both $\mathrm{Cr}$ absorbers, i.e. $\mathrm{Cr}^{3+}$ and $\mathrm{Cr}^{4+}$. Furthermore it was found that $(69 \pm 1) \%$ of the $\mathrm{Cr}$ ions are in the trivalent state and the remaining $(31 \pm 1) \%$ in the tetravalent state (the exact values of all fitting parameters are tabulated in Table 3 with their respective errors). The $R$ factor of the fit was in this case well below 0.020 . The resulting fit is shown in Fig. 4 and compared to the experimental LGO:Cr EXAFS spectrum.

All the spectra are similar within the error margins, so no direct indication for the presence of $\mathrm{M}(\mathrm{rv})$ ions in the direct neighbourhood (within $4.5 \AA$ from the absorber) could be found for the codoped samples. There are two possible reasons for this phenomenon: (1) there is no incorporation of $\mathrm{M}(\mathrm{rv})$ in the direct neighbourhood of the Cr ions, or (2) the similar atomic scattering factors of Ge and Ga do not allow us to differentiate between them.

Besides the preferential substitution of the $\mathrm{Cr}$ ions on the octahedral sites, it is also observed that the $\mathrm{Cr}-\mathrm{O}$ distance is smaller than the Ga-O distance. This effect can be caused by the shorter natural bond length for $\mathrm{Cr}-\mathrm{O}$ bonds (e.g. in $\mathrm{Cr}_{2} \mathrm{O}_{3}$ and $\mathrm{CrO}_{2}$ ) compared to the $\mathrm{Ga}-\mathrm{O}$ bond.

\section{Electron paramagnetic resonance}

Powder EPR spectra were measured both at X-band $(9.42 \mathrm{GHz})$ and Q-band (34.0 GHz) frequencies (Fig. 5). Spin Hamiltonian parameters for $\mathrm{Cr}^{3+}$ in LGO were extracted by simultaneous simulation of the X- and Q-band spectra with identical parameters, using Easyspin in Matlab. ${ }^{34}$

The following effective spin Hamiltonian for the $\mathrm{Cr}^{3+}$ ion ( $S=3 / 2$, ground state ${ }^{4} \mathrm{~A}_{2}$ ), consisting of a Zeeman and a zerofield splitting (ZFS) term, was used: $:^{35,36}$

$$
\begin{aligned}
\hat{H}_{\mathrm{S}} & =\hat{H}_{\text {Zeeman }}+\hat{H}_{\text {ZFS }} \\
& =g \mu_{\mathrm{B}} \vec{B} \cdot \hat{\vec{S}}+\frac{D}{3}\left(3 \hat{S}_{z}^{2}-\hat{S}^{2}\right)+E\left(\hat{S}_{x}^{2}-\hat{S}_{y}^{2}\right)
\end{aligned}
$$

where $\mu_{\mathrm{B}}$ is the Bohr magneton, the $g$ tensor was assumed to be isotropic, and $D$ and $E$ represent ZFS parameters.

The simulated spectra and the values of the ZFS parameters point to $\mathrm{Cr}^{3+}$ being present at rhombically distorted octahedral 

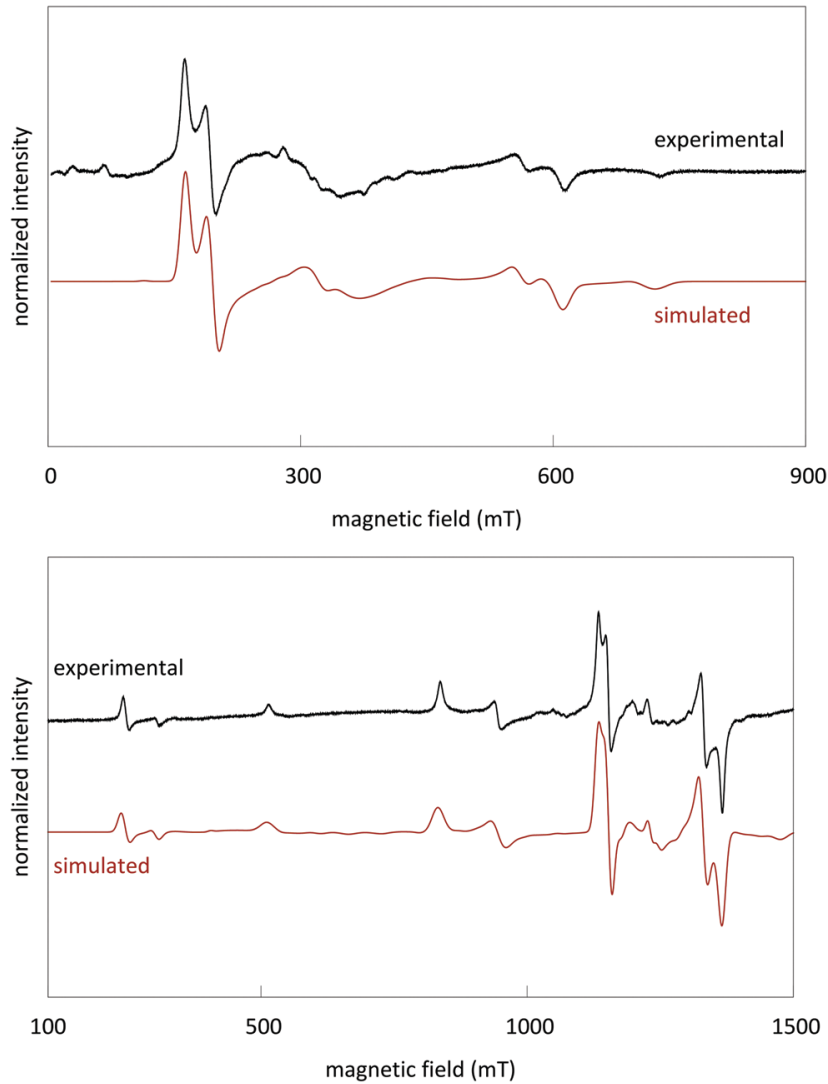

Fig. 5 Experimental and simulated X-band (top) and Q-band (bottom) spectra of LGO:Cr. The EPR spectra were simulated using (1) a spin 3/2 system for $\mathrm{Cr}^{3+}$ with spin Hamiltonian parameters as detailed in the text, and (2) a system with effective spin 1/2 to account for the broad component around $g=1.99$.

sites. This is in accordance with the results from XAS. The values for $g, D$ and $E$ are determined as

$$
\begin{gathered}
g=1.98 \pm 0.01 \\
D=0.332 \pm 0.005 \mathrm{~cm}^{-1} \\
E / D=0.057 \pm 0.009
\end{gathered}
$$

which correspond to the values for the $\mathrm{Cr}^{3+}$ ion in LGO and related spinels, found in the literature. ${ }^{37,38}$ Experimentally, anisotropy in $g$ could not be resolved. Because the measurements are done over such a wide magnetic field range, the simulations are less sensitive to the $g$ value, and as such larger errors on $g$ are determined.

$D$ arises due to an axial deviation from cubic symmetry along the $\langle 111\rangle$ direction of the unit cell. In regular spinel, only this axial component is present and will contribute to the EPR spectrum of $\mathrm{Cr}^{3+}$. However, in LGO, the partial occupation of octahedral lattice sites by $\mathrm{Li}^{+}$ions in the second nearest neighbor cation shell causes a reduction of the trigonal $D_{3 \mathrm{~d}}$ local symmetry (found in regular spinel) to the rhombic $C_{2}$ symmetry. This is the origin of the small rhombic ZFS parameter $E$. The $\mathrm{Cr}^{3+}$ ground state is split into two Kramers doublets.
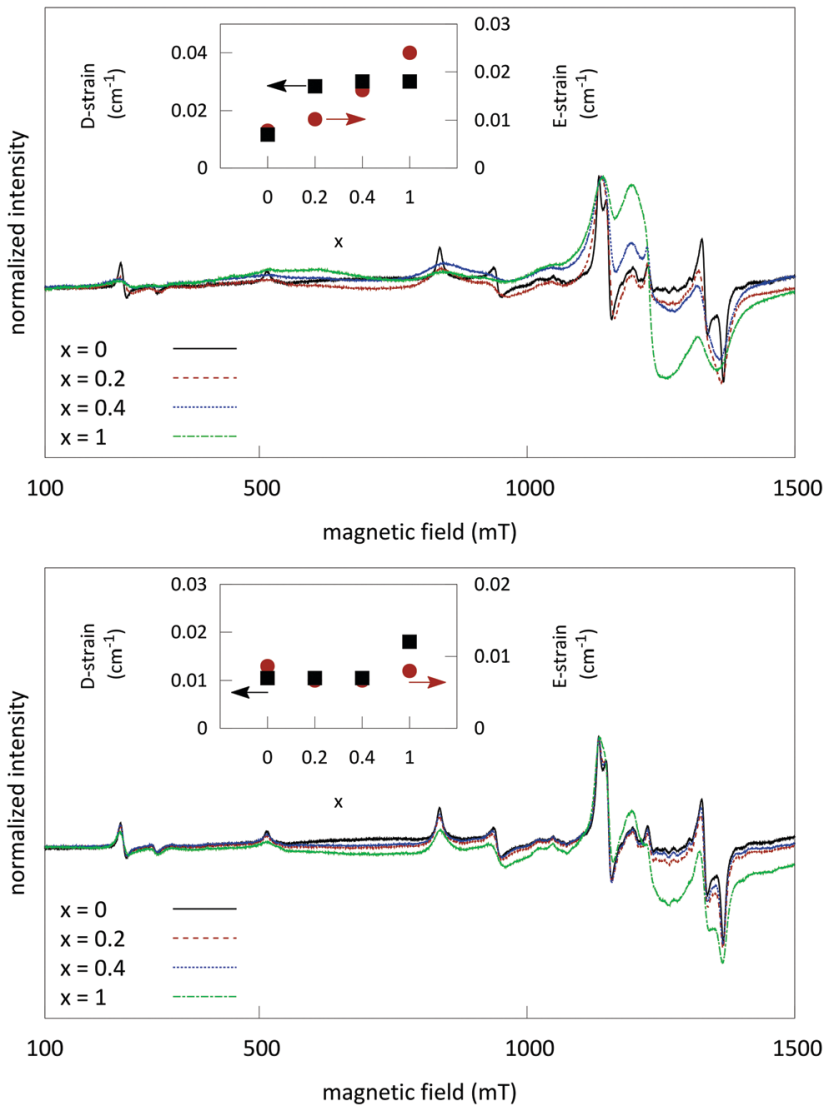

Fig. 6 Normalized EPR spectra of LGO:Ge,Cr (top) and LGO:Si,Cr (bottom) with varying values of $x$. The spectra show an increase in EPR linewidth and an increase of the prominent line around $g=1.99$, when increasing the $\mathrm{M}(\mathrm{IV})$ content. All spectra were recorded at $\mathrm{RT}$ at the $\mathrm{Q}$-band frequency $(34.0 \mathrm{GHz})$. Insets: Values for the $D$ and $E$ strains for $L G O: C r, M$ samples. These were obtained from simultaneous simulation of $X$ - and $Q$-band data, while keeping the determined $g, D$ and $E$ parameters fixed.

The total ZFS between the Kramers doublets at $B=0 \mathrm{mT}$ is $2\left(D^{2}+3 E^{2}\right)^{1 / 2}=0.665 \mathrm{~cm}^{-1} \cdot 37$

Fig. 6 shows an overlay of the EPR spectra of LGO:Ge,Cr and LGO:Si,Cr for varying values of $x$. The results show a variation in experimental EPR linewidth, depending on $x$. The linewidths can be modeled by strains on the ZFS parameters (shown in the insets of Fig. 6). These strains reflect slightly different surroundings of the $\mathrm{Cr}^{3+}$ ions, experiencing varying magnitudes in crystal field.

At $x=1$ for LGO:Ge,Cr, a broad feature becomes prominent around $1200 \mathrm{mT}$, corresponding to an effective $g$-value of 1.99, which could be attributed to Cr clustering and interaction between Cr ions via dipole-dipole exchange coupling or $\mathrm{Cr}$ incorporation in $\mathrm{GeO}_{2}$ side phases. ${ }^{18,39,40}$ For the LGO:Si,Cr samples, the same feature around $g=1.99$ becomes prominent, but the linewidths do not vary as much between the other samples, thus implying that introducing $\mathrm{Si}^{4+}$ does not lead to strong distortion of the $\mathrm{Cr}^{3+}$ sites.

Different LGO:Cr samples show a small distribution in the EPR linewidth. This reflects for instance slight variations in local Cr content or a different distribution of surrounding defects between samples. 


\section{Photoluminescence and afterglow}

Fig. 7 shows the PL excitation and emission spectrum for $\mathrm{Cr}^{3+}$-doped LGO samples. The emission profile centers around a sharp line at $718 \mathrm{~nm}$. This line corresponds to the zerophonon emission of $\mathrm{Cr}^{3+}$ from the ${ }^{2} \mathrm{E}$ level to the ${ }^{4} \mathrm{~A}_{2}$ ground state (known as the $\mathrm{R}$ transition ${ }^{41}$ ). The broad underlying feature from $650 \mathrm{~nm}$ to $750 \mathrm{~nm}$ corresponds to the ${ }^{4} \mathrm{~T}_{2} \rightarrow{ }^{4} \mathrm{~A}_{2}$ spin allowed transition. R1 phonon side bands (both Stokes
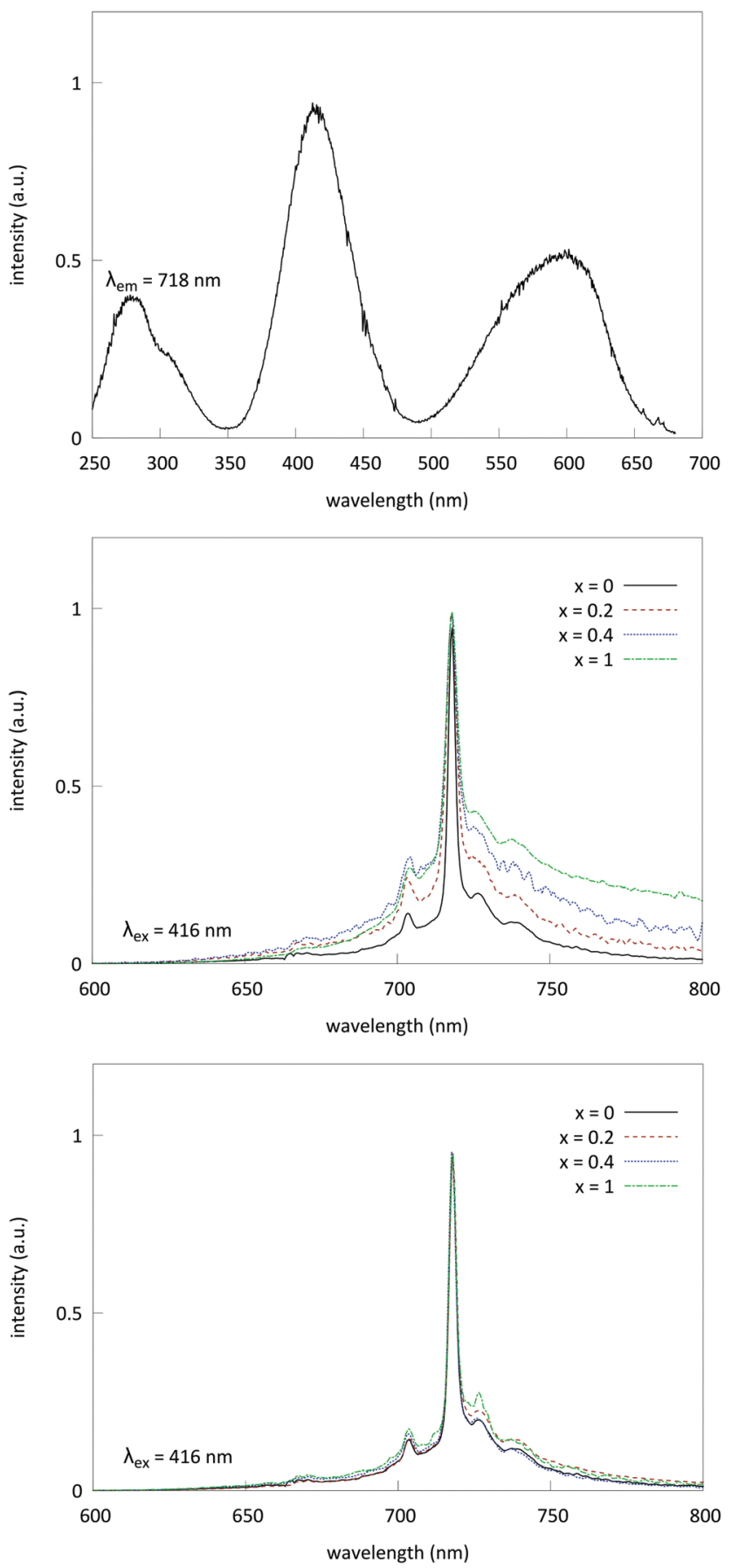

Fig. 7 Normalized steady state excitation (top) and emission of LGO:Ge, $\mathrm{Cr}$ (middle) and LGO:Si,Cr (bottom) for varying values of $x$. Excitation was monitored at $718 \mathrm{~nm}$, and emission was recorded upon excitation at $416 \mathrm{~nm}$.

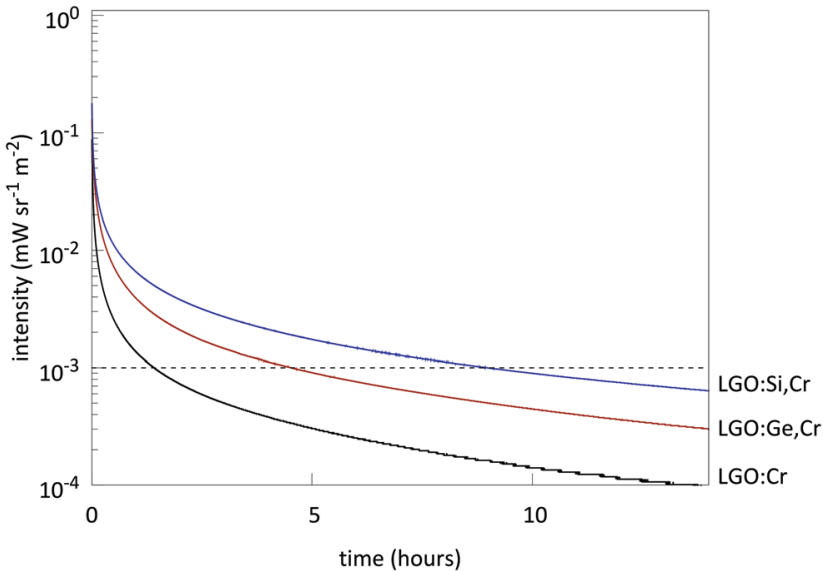

Fig. 8 Afterglow profiles of LGO: $\mathrm{Cr}$ (black), LGO:Si,Cr (blue) and LGO:Ge, $\mathrm{Cr}$ (red). $x=0.4$ for M(iv)-doped samples. The dashed line represents the threshold value for determination of the powder afterglow time.

and anti-Stokes) are situated on top of the broad emission band. Separation of the phonon side bands is of the order of $200-400 \mathrm{~cm}^{-1}$, which is well in the range of phonon frequencies of LGO, as reported by Keramidas et al. ${ }^{42}$

As the Ge content is increased in the samples, the local disorder around the $\mathrm{Cr}^{3+}$ ions increases and consequently the $R$-line broadens and, proportionally, broad band emission from the ${ }^{4} \mathrm{~T}_{2}$ state increases in intensity. These results are in line with the EPR data that show significant broadening of the $\mathrm{Cr}^{3+}$ EPR signal, due to incorporation of Ge. In samples codoped with $\mathrm{Si}$, there is no significant change in the emission profile of chromium, which is again in line with the results obtained from EPR.

Excitation bands arise at $280 \mathrm{~nm}, 410 \mathrm{~nm}\left({ }^{4} \mathrm{~A}_{2} \rightarrow{ }^{4} \mathrm{~T}_{1}\left[{ }^{4} \mathrm{~F}_{2}\right]\right.$ transition) and $600 \mathrm{~nm}\left({ }^{4} \mathrm{~A}_{2} \rightarrow{ }^{4} \mathrm{~T}_{2}\right.$ transition $)$, when monitoring the emission at $718 \mathrm{~nm}$. The excitation band at $280 \mathrm{~nm}$ can be attributed to either the ${ }^{4} \mathrm{~A}_{2} \rightarrow{ }^{4} \mathrm{~T}_{1}\left[{ }^{4} \mathrm{P}\right]$ transition or possibly charge-transfer from $\mathrm{Cr}^{3+}$ to a nearby oxygen. The optical bandgap of undoped LGO (derived from diffuse reflectance measurements, not shown) is about $4.9 \mathrm{eV}$. Low temperature measurements show no reduction of intensity of the excitation band in the UV, which indicates a higher contribution of $\mathrm{d}-\mathrm{d}$ transitions compared to charge transfer.

No afterglow could be induced by excitation in the visible range. Only excitation in the lowest excitation band around $280 \mathrm{~nm}$ induces persistent luminescence in LGO.

Measuring afterglow in radiometric units provides useful comparisons between persistent phosphors. ${ }^{2,4}$ Afterglow for LGO:Cr could be measured for over $1 \mathrm{~h}$ (Fig. 8), before the intensity drops below $10^{-3} \mathrm{~mW} \mathrm{sr}^{-1} \mathrm{~m}^{-2}$. Afterglow is enhanced significantly by the addition of $\mathrm{M}$ (Iv) to the host (Fig. 9). The optimal nominal $x$-value for the afterglow is $x=0.4$ according to the formula $\mathrm{LiGa}_{5-x} \mathrm{M}_{x} \mathrm{O}_{8}: \mathrm{Cr}^{3+}$. If $x$ exceeds the nominal $x=1$ value, both PL and afterglow intensity decrease in comparison to the non-codoped samples.

\section{Discussion}

The results obtained from XAS, EPR and PL all point to $\mathrm{Cr}$ being incorporated in the octahedral sites of the LGO lattice. 


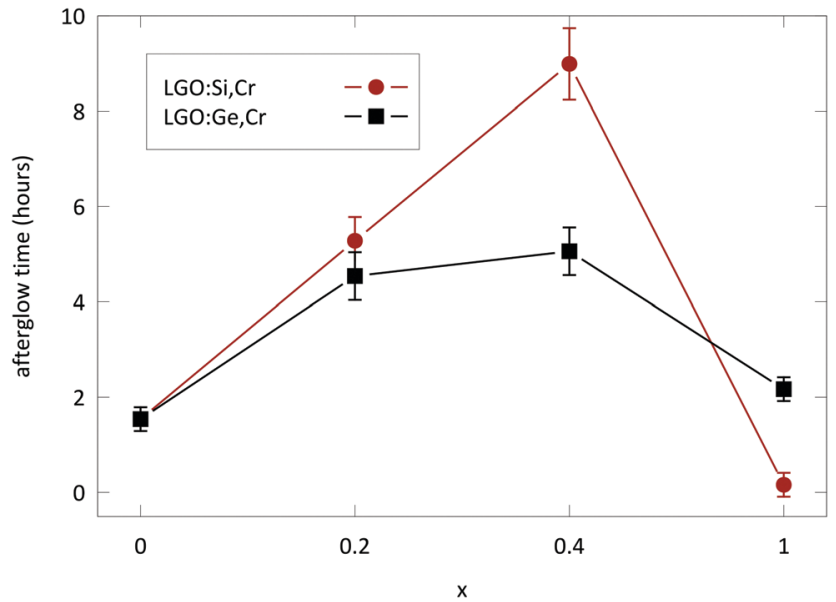

Fig. 9 Afterglow time of the LGO:Cr,M samples with varying $M(\mathrm{IV})$ content. The afterglow time is defined as the time it takes for the intensity to drop below $10^{-3} \mathrm{~mW} \mathrm{sr}^{-1} \mathrm{~m}^{-2}$. Error bars are derived from four consecutive measurements of the same sample.

The afterglow intensity significantly increases due to codoping with $\mathrm{Si}$ and Ge, but only Ge seems to induce strong additional disorder around the $\mathrm{Cr}$ site.

Seeing how the strains on the ZFS parameters $D$ and $E$ are intrinsically linked to disorder along the axial $\langle 111\rangle$ direction (tetrahedral sites) and rhombic $\langle 110\rangle$ direction (octahedral sites), respectively, ${ }^{37}$ the significant effect of Ge codoping on both the $D$-strain and $E$-strain of the Cr EPR signal could indicate that $\mathrm{Ge}$ is stochastically incorporated on both tetrahedral and octahedral sites. ${ }^{18}$ The small measured effect of $\mathrm{Si}$, on the other hand, indicates that it is mainly incorporated on octahedral sites without much structural distortion. However, since Si codoping has the largest effect on the afterglow and $\mathrm{M}(\mathrm{Iv})$ incorporation is limited, it seems that $\mathrm{M}$ (rv) incorporation indirectly induces additional host defects in the LGO lattice.

From XAS measurements, it is clear that $\mathrm{Cr}$ is present in both trivalent and tetravalent state. During the collection of the XAS spectra, no significant reduction of the Cr intensity or shift in absorption edge was found. This indicates that no valence changes of $\mathrm{Cr}$ are induced by the X-ray irradiation itself. Additional radioluminescence measurements, utilizing full spectrum $\mathrm{Cu}$-anode $\mathrm{X}$-ray excitation, also did not show any appreciable degradation of the $\mathrm{Cr}^{3+}$ intensity over the course of several hours (Fig. S10, ESI $\dagger$ ). The presence of $\mathrm{Cr}^{4+}$ could not be verified via any of the other employed experimental techniques. $\mathrm{Cr}^{4+}$ has a spin state of $S=1$; in low-symmetry environments, it is not evident to detect EPR signal for such a system. Optical transitions of $\mathrm{Cr}^{4+}$ were not detected by diffuse reflectance measurements (Fig. S9, ESI $\dagger$ ).

Nonetheless, tetravalent chromium is clearly present in the LGO lattice. There is thus a charge mismatch between the $\mathrm{Cr}^{4+}$ species and the $3+$ charge of the Ga sites, which means that chromium doping induces positive point defects at the doping sites, which need to be compensated by additional defects. In this way, Cr itself is partly responsible for the persistent behavior of the LGO phosphor. Codoping with M(rv), then, will only enhance the number of positive point defects and thus cause an increase of the persistent luminescence. Furthermore, tetravalent chromium can possibly act as an electron trap during the charging of the persistent phosphor. ${ }^{43,44}$

Additionally, concentration quenching and increase of $\mathrm{Cr}$ clusters with the concentration will impede the persistent behavior, whereas codoping with $\mathrm{M}(\mathrm{Iv})$ ions only leads to the increase of traps and not to the reduction of PL properties.

\section{Conclusions}

We successfully prepared the NIR persistent phosphor $\mathrm{LiGa}_{5} \mathrm{O}_{8}: \mathrm{Cr}^{3+}$ and were able to improve the persistent behavior by codoping with $\mathrm{M}(\mathrm{rv})$ ions ( $\mathrm{M}=\mathrm{Si}, \mathrm{Ge})$, according to the formula $\mathrm{LiGa}_{5-x} \mathrm{M}_{x} \mathrm{O}_{8}: \mathrm{Cr}^{3+}$ $(x=0.2,0.4$ or 1$)$. The afterglow profile is strongest for $x=0.4$, for both Si and Ge. From SEM-EDX measurements it is clear that there is a discrepancy between nominal and actual $\mathrm{M}$ (Iv) content. The improvement in persistent behavior is thus likely related to the occurrence of charge compensation effects during the synthesis.

The local environment of $\mathrm{Cr}^{3+}$ was investigated via XAS and EPR. Both types of measurements agree that $\mathrm{Cr}^{3+}$ is found on rhombically distorted octahedral sites in the LGO lattice. The Cr EPR signal is significantly broadened by the inclusion of $\mathrm{Ge}^{4+}$, revealing the additional disorder around $\mathrm{Cr}$ due to codoping. The increased local disorder is also reflected in the broadening of the emission peaks in the PL spectra.

XAS measurements show that chromium is present in the host lattice in two valence states: III and IV. Tetravalent chromium will induce charge compensation point defects and possibly act as electron trap during the charging of the persistent phosphor.

\section{Conflicts of interest}

There are no conflicts to declare.

\section{Acknowledgements}

O. Q. De Clercq and J. Kusakovskij gratefully acknowledge the financial support of the Ghent University's Special Research Fund (BOF). The authors would like to acknowledge Dipanjan Banerjee and the rest of the staff at DUBBLE for experimental support and useful discussions.

\section{References}

1 T. Matsuzawa, Y. Aoki, N. Takeuchi and Y. Murayama, J. Electrochem. Soc., 1996, 143, 2670-2673.

2 K. Van den Eeckhout, P. F. Smet and D. Poelman, Materials, 2010, 3, 2536-2566.

3 K. Van den Eeckhout, D. Poelman and P. F. Smet, Materials, 2013, 6, 2789-2818.

4 P. F. Smet, K. Van den Eeckhout, O. Q. De Clercq and D. Poelman, in Handbook on the Physics and Chemistry of Rare Earths, ed. B. Jean-Claude and K. P. Vitalij, Elsevier, 2015, vol. 48, pp. 1-108. 
5 Q. le Masne de Chermont, C. Chaneac, J. Seguin, F. Pelle, S. Maitrejean, J. P. Jolivet, D. Gourier, M. Bessodes and D. Scherman, Proc. Natl. Acad. Sci. U. S. A., 2007, 104, 9266-9271. 6 J. Frangioni, Curr. Opin. Chem. Biol., 2003, 7, 626-634.

7 Y. Zhuang, Y. Katayama, J. Ueda and S. Tanabe, Adv. Opt. Mater., 2014, 36, 1907-1912.

8 B. Viana, S. K. Sharma, D. Gourier, T. Maldiney, E. Teston, D. Scherman and C. Richard, J. Lumin., 2016, 170, 879-887.

9 R. Wang and F. Zhang, J. Mater. Chem. B, 2014, 2, 2422.

10 S. K. Singh, RSC Adv., 2014, 4, 58674-58698.

11 K. Van den Eeckhout, P. F. Smet and D. Poelman, J. Lumin., 2009, 129, 1140-1143.

12 T. Maldiney, G. Sraiki, B. Viana, D. Gourier, C. Richard, D. Scherman, M. Bessodes, K. Van den Eeckhout, D. Poelman and P. F. Smet, Opt. Mater. Express, 2012, 2, 261-268.

13 A. Bessière, S. Jacquart, K. Priolkar, A. Lecointre, B. Viana and D. Gourier, Opt. Express, 2011, 19, 10131-10137.

14 M. Allix, S. Chenu, E. Véron, T. Poumeyrol, E. A. KouadriBoudjelthia, S. Alahraché, F. Porcher, D. Massiot and F. Fayon, Chem. Mater., 2013, 25, 1600-1606.

15 Z. Pan, Y. Y. Lu and F. Liu, Nat. Mater., 2011, 11, 58-63.

16 F. Shen, C. Deng, X. Wang and C. Zhang, Mater. Lett., 2016, 178, 185-189.

17 F. Shen, C. Deng, X. Wang, C. Zhang and W. Liu, J. Mater. Sci.: Mater. Electron., 2016, 27, 9067-9072.

18 D. Gourier, A. Bessière, S. K. Sharma, L. Binet, B. Viana, N. Basavaraju and K. R. Priolkar, J. Phys. Chem. Solids, 2014, 75, 826-837.

19 L. Binet, S. K. Sharma and D. Gourier, J. Phys.: Condens. Matter, 2016, 28, 385501.

20 S. K. Sharma, A. Bessière, N. Basavaraju, K. R. Priolkar, L. Binet, B. Viana and D. Gourier, J. Lumin., 2014, 155, 251-256.

21 N. Basavaraju, K. R. Priolkar, A. Bessiere, S. K. Sharma, D. Gourier, L. Binet, B. Viana and S. Emura, Phys. Chem. Chem. Phys., 2017, 19, 1369-1377.

22 F. Liu, W. Yan, Y. J. Chuang, Z. Zhen, J. Xie and Z. Pan, Sci. Rep., 2013, 3, 1554.

23 X. Fu, C. Liu, J. Shi, H. Man, J. Xu and H. Zhang, Adv. Opt. Mater., 2014, 36, 1792-1797.
24 M. Borsboom, W. Bras, I. Cerjak, D. Detollenaere, D. Glastra van Loon, P. Goedtkindt, M. Konijnenburg, P. Lassing, Y. K. Levine, B. Munneke, M. Oversluizen, R. van Tol and E. Vlieg, J. Synchrotron Radiat., 1998, 5, 518-520.

25 W. Bras, S. Nikitenko, G. Portale, A. Beale, A. V. D. Eerden and D. Detollenaere, J. Phys.: Conf. Ser., 2010, 247, 012047.

26 B. Ravel and M. Newville, J. Synchrotron Radiat., 2005, 12, 537-541.

27 R. Shannon, Acta Crystallogr., Sect. A: Cryst. Phys., Diffr., Theor. Gen. Crystallogr., 1976, 32, 751-767.

28 B. H. O'Connor, D. Y. Li and B. A. Hunter, Adv. X-Ray Anal., 2001, 44, 96-102.

29 J. Ahman, G. Svensson and J. Albertsson, Acta Chem. Scand., 1996, 50, 391-394.

30 K. E. Sickafus and J. M. Wills, J. Am. Ceram. Soc., 1999, 82, 3279-3292.

31 R. K. Datta, J. Am. Ceram. Soc., 1971, 54, 262.

32 V. Singh, R. V. S. S. N. Ravikumar, G. Sivaramaiah, J. L. Rao and S. H. Kim, Mater. Res. Bull., 2015, 61, 183-188.

33 F. M. F. de Groot, J. Electron Spectrosc. Relat. Phenom., 1994, 67, 529-622.

34 S. Stoll and A. Schweiger, J. Magn. Reson., 2006, 178, 42-55.

35 A. Abraham and B. Bleaney, Electron Paramagnetic Resonance of Transition Ions, Oxford University Press, 2 edn, 2012.

36 C. Rudowicz and S. K. Misra, Appl. Spectrosc. Rev., 2001, 36, 11-63.

37 T. J. Glynn, J. Chem. Phys., 1971, 55, 2925.

38 Y. Mei, C. Liu and W.-C. Zheng, Optik, 2017, 131, 183-187.

39 B. V. Padlyak and A. Gutsze, Appl. Magn. Reson., 1998, 14, 59-68.

40 R. J. Landry, J. T. Fournier and C. G. Young, J. Chem. Phys., 1967, 46, 1285-1290.

41 H. Szymczak, M. Warddzynska and I. E. Mylnikova, J. Phys. C: Solid State Phys., 1975, 8, 3937-3943.

42 V. G. Keramidas, B. A. Deangelis and W. B. White, J. Solid State Chem., 1975, 15, 233-245.

43 Y. Zhuang, J. Ueda and S. Tanabe, Appl. Phys. Express, 2013, 6, 052602.

44 J. Xu, J. Ueda, Y. Zhuang, B. Viana and S. Tanabe, Appl. Phys. Express, 2015, 8, 042602. 